\title{
NÚMERO DE ANOS EM TRATAMENTO DIALÍTICO CRÔNICO E RISCO DE MORTE EM PACIENTES COM E SEM DIABETES MELITO
}

\author{
Antonio Alberto Lopes*, Paulo Benigno Pena Batista, Fernando Antonio Costa, \\ Miriam M. Nery, Gildete Barreto Lopes \\ Trabalho realizado no Hospital São Rafael, Salvador, BA.
}

RESUMO - OBjetivos. Avaliar se, no Brasil, o número de anos em tratamento dialítico dos pacientes portadores de estágio final de doença renal (EFDR), com e sem diabetes melito, está relacionado com o risco de morte.

Métodos. Análise de dados do Ministério da Saúde do Brasil de 25.952 pacientes com idade superior a 18 anos, portadores de EFDR, que estavam em tratamento dialítico em abril de 1997. Foram registrados os casos de óbito que ocorreram entre abril de 1997 e julho de 2000. Modelos de Cox foram utilizados para estimar o risco relativo (RR) da associação entre número de anos em tratamento dialítico e risco de morte.

Resultados. 0 risco de morte foi significantemente menor nos pacientes que estavam em diálise entre 3 e $<10$ anos, comparado com I e $<2$ anos (RR entre 0,78 e 0,88). Com o ajuste para idade e diabetes, este efeito do número de anos em diálise reduziu-se. Em diabéticos $(n=2.952), 0$ risco ajustado de morte mostrou uma tendência de redução com o aumento do número de anos em diálise.

Conclusöes. No Brasil, o risco de morte de pacientes portadores de EFDR reduz-se com 0 aumento do número de anos em tratamento dialítico, até aproximadamente 10 anos. Este achado pode ser parcialmente explicado pela menor média de idade e pela menor prevalência de diabetes nos que estavam em diálise por um tempo mais longo. 0 efeito do número de anos em tratamento dialítico sobre o risco de morte parece ser mais forte em diabéticos.

UNITERMOS: Diálise. Risco de morte. Estágio final de doença renal.

\section{INTRODUÇÃO}

Apesar do progresso na área de terapia renal de substituição (diálise ou transplante renal), a expectativa de vida de pacientes em estágio final de doença renal (EFDR) é muito menor do que a descrita nos diversos grupos da mesma faixa etária na população geral ${ }^{1,2} .0$ risco de morte em pacientes em EFDR é particularmente elevado em diabéticos, em brancos e em idosos ${ }^{1,3}$. Tem sido também mostrado que o número de anos que o paciente permanece em tratamento dialítico está relacionado com o risco de morte ${ }^{4-6}$. Portanto, o número de anos em diálise pode ajudar a estimar a probabilidade de um paciente com EFDR permanecer vivo por um certo período de tempo, caso não seja possível oferecer-lhe um transplante renal.

Nos Estados Unidos, Chertow et al. estimaram que o risco de morte aumenta em $6 \%$ para cada ano adicional em diálise, após ajuste para os efeitos de diversas co-variáveis ${ }^{5}$. 0

* Correspondência:

Rua Mar. Floriano 448 apt 1301, Canela 40110-010 - Salvador - BA

Telefone: 071-336-1558 - Fax: 071-213-6710 aaslopes@ufba.br estudo de Chertow et al., no entanto, não permite assegurar se o risco se reduz ou se estabiliza depois de um certo período. Em verdade, eles não deixaram claro se a associação entre tempo em tratamento dialítico e risco de morte é linear. Não se pode também assegurar se os resultados descritos, em pacientes com EFDR, tratados nos Estados Unidos, podem ser generalizados para outros locais. O presente trabalho foi desenvolvido para avaliar se existe relação entre número de anos em diálise e risco de morte em pacientes adultos com EFDR, no Brasil, levando em consideração a idade, o sexo, a região geográfica e o diagnóstico de diabetes melito.

\section{Métodos}

Os dados dos pacientes com EFDR, tratados através diálise nos diversos centros do Brasil, em abril de 1997, foram fornecidos pelo Ministério da Saúde (sistema "Autorização de Procedimentos de Alta Complexidade/ Custo", APAC). A análise foi restrita aos que tinham idade superior a 18 anos, em abril de 1997. As seguintes variáveis foram consideradas na análise: data de nascimento, data do óbito, data do primeiro transplante, data de transferência para transplante, sexo, presença de código internacional de doenças (CID) indicando diabetes melito, e região geográfica onde o paciente estava sendo dialisado. Foram excluídos da análise pacientes submetidos a transplante renal antes de abril de 1997. Os pacientes foram acompanhados até julho de 2000 ou até a data do óbito, do abandono do tratamento dialítico ou da transferência para transplante.

A análise de variância (ANOVA) e o teste do qui-quadrado foram utilizados para comparar médias e proporções, respectivamente. $O$ risco relativo (RR) de morte foi estimado através de modelos de risco proporcional (regressão de (ox), utilizando-se, como grupo de referência, os pacientes que estavam em diálise por um período de I a menos de 2 anos (I-<2anos). Quatro modelos foram utilizados para avaliar-se a associação entre o número de anos em tratamento dialítico e o risco de morte: I) não ajustado, 2) ajustado para idade no inicio do estudo, 3) ajustado para idade, sexo e região geográfica e 4) ajustado para diabetes e as variáveis incluídas no modelo 3 . Foram também realizadas análises específicas para diabéticos e não diabéticos, ajustadas para idade, sexo e região geográfica. As análises foram feitas utilizando o software "Statistical 


\begin{tabular}{|c|c|c|c|c|c|c|c|c|c|}
\hline & \multicolumn{9}{|c|}{ Anos em Tratamento Dialítico* } \\
\hline & $\begin{array}{c}\leq 1 \\
n=8705\end{array}$ & $\begin{array}{c}\mathrm{I}-<2 \\
N=5389\end{array}$ & $\begin{array}{c}2-<3 \\
n=3507\end{array}$ & $\begin{array}{c}3-<4 \\
n=2561\end{array}$ & $\begin{array}{c}4-<5 \\
n=1693\end{array}$ & $\begin{array}{c}5-<7 \\
n=2130\end{array}$ & $\begin{array}{c}7-<10 \\
n=1322\end{array}$ & $\begin{array}{c}\geq 10 \\
n=645\end{array}$ & $\begin{array}{c}\text { Total } \\
n=25952\end{array}$ \\
\hline Idade (média $\pm D P$ ) *** & $50,0 \pm 15,3$ & $49,2 \pm 15,0$ & $48,9 \pm 14,6$ & $48,1 \pm 14,6$ & $48,0 \pm 14,6$ & $47,7 \pm 13,9$ & $46,9 \pm 13,3$ & $46,7 \pm 12,5$ & $48,9 \pm 14,8$ \\
\hline$\%$ Masculino & 58,6 & 59,0 & 56,8 & 56,2 & 58,0 & 54,8 & 53,8 & 54,9 & 57,5 \\
\hline$\%$ Diabetes & 15,7 & 13,2 & 10,3 & 8,7 & 7,4 & 5,1 & 2,8 & 1,9 & $1 \mathrm{I}, 4$ \\
\hline \multicolumn{10}{|l|}{ \% Região } \\
\hline Norte & 1,1 & 0,7 & 0,9 & 1,2 & 1,4 & 1,0 & 0,2 & 0 & 1,0 \\
\hline Nordeste & 18,1 & $|8|$, & 17,3 & 19,8 & 21,7 & 22,0 & 22,8 & 27,3 & 19,2 \\
\hline Centro-Oeste & 7,3 & 6,8 & 5,5 & 3,5 & 3,7 & 2,9 & 2,4 & I,4 & 5,6 \\
\hline Sudeste & 56,2 & 57,6 & 59,3 & 59,4 & 58,1 & 59,7 & 59,1 & 56,7 & 57,8 \\
\hline Sul & 17,3 & 16,8 & 17,0 & $|6|$, & 15,2 & $\mid 4,4$ & 15,5 & 14,6 & 16,5 \\
\hline
\end{tabular}

* Todas as comparações entre número de anos em tratamento dialítico e características dos pacientes foram estatisticamente significantes $(p<0,00 \mathrm{I})$

** idade em abril de 1997

Tabela 2 - Associações não-ajustadas e ajustadas através da regressão de Cox da associação entre número de anos em tratamento dialítico e risco de morte

\begin{tabular}{|c|c|c|c|c|}
\hline \multirow[b]{3}{*}{$\begin{array}{c}\text { Anos em tratamento } \\
\text { dialítico }\end{array}$} & \multicolumn{4}{|c|}{ Risco relativo } \\
\hline & \multirow[b]{2}{*}{ Não-ajustado } & \multicolumn{3}{|c|}{ Ajustado } \\
\hline & & Idade & $\begin{array}{c}\text { Idade, Sexo } \\
\text { e RG }\end{array}$ & $\begin{array}{c}\text { Idade, Sexo, } \\
\text { RG e DM }\end{array}$ \\
\hline$\leq 1$ & $\mathrm{I}, 14^{*}$ & $1,12 *$ & $1,12^{*}$ & 1,10 \\
\hline $\mid-<2$ & $\operatorname{Ref}=1$ & $\operatorname{Ref}=1$ & $\operatorname{Ref}=1$ & $\operatorname{Ref}=1$ \\
\hline $2-<3$ & 0,96 & 0,98 & 0,97 & 1,00 \\
\hline $3-<4$ & $0,88 *$ & $0,92 * *$ & $0,92 * *$ & 0,95 \\
\hline $4-<5$ & $0,85 *$ & $0,90 * *$ & $0,90 * *$ & 0,95 \\
\hline $5-<7$ & $0,87 *$ & $0,92 * *$ & $0,92 * *$ & 0,97 \\
\hline $7-<10$ & $0,78 *$ & $0,86 *$ & $0,86 *$ & 0,92 \\
\hline$\geq 10$ & 0,90 & 1,02 & 1,02 & I,II \\
\hline
\end{tabular}

Ref = referência; $R G$ = região geográfica; $D M=$ diabetes melito;

* $p<0,05 ; * * 0,1>p>0,05$

Package for Social Science" (SPSS), versão para Windows ${ }^{7}$. Valores de $p<0,05$ foram considerados estatisticamente significantes.

\section{Resultados}

Foram estudados 25.952 pacientes. A média de idade dos pacientes, em abril de 1997, era 48,9 $\pm 14,8$ anos. O sexo masculino correspondeu a $57,5 \%(n=14.932)$ da amostra. A prevalência de diabetes melito foi estimada em II,4\% $(n=2.952)$. Os percentuais de pacientes, sendo dialisados nas regiões Norte, Nordeste, Centro-oeste, Sudeste e Sul foram, respectivamente, $1 \%, 19,2 \%$, $5,6 \%, 57,8 \%$ e $16,5 \%$.

A Tabela I mostra as características dos pacientes, de acordo com o tempo em diálise. Os grupos com tempo maior em diálise apre- sentaram menor media de idade e menor prevalência de diabetes $(\mathrm{p}<0,00 \mathrm{I})$. A predominância do sexo masculino foi mais notória nos grupos com menor tempo em diálise $(p<0,00 \mathrm{I})$. A região geográfica foi também significantemente $(p<0,00 \mathrm{I})$ associada como número de anos em diálise, sendo observado um aumento do percentual de pacientes da região nordeste, à medida que aumentava 0 número de anos em diálise.

A Tabela 2 mostra as estimativas do RR de morte, de acordo com o número de anos em diálise, utilizando-se $1-<2$ anos como referência. No modelo não ajustado, o risco de morte foi significantemente maior nos pacientes tratados por tempo inferior a um ano, na época em que se iniciou o estudo $(R R=I, I 4$; $\mathrm{p}<0,05)$. $\mathrm{O}$ risco de morte não foi signifi- cantemente diferente entre o grupo de pacientes tratados por $2-<3$ anos e o grupo de referência ( $\mathrm{I}-<2$ anos). O risco de morte foi, no entanto, significantemente $(p<0,05)$ menor em pacientes que estavam em tratamento entre $3 \mathrm{e}<10$ anos do que entre $\mathrm{I} \mathrm{e}<2$ anos (RR entre 0,79 e 0,88 ).

A associação entre número de anos em tratamento dialítico e risco de morte tornouse menos acentuada após o ajuste para idade, sendo pouco alterada com o ajuste para sexo e região geográfica. $O$ efeito de anos em diálise sobre o risco de morte, no entanto, foi reduzido após a inclusão de diabetes melito no modelo de Cox. Um risco significantemente maior de morte foi observado em pacientes que estavam em tratamento por tempo menor do que um ano, mesmo após o ajuste para todas as co-variáveis (i.e., idade, sexo, região geográfica e diabetes melito). Os resultados observados no modelo não ajustado e no ajustado são também mostrados na Figura I.

A Figura 2 mostra os riscos relativos de morte ajustados para as variáveis demográficas, separadamente para os diabéticos e os não diabéticos. Devido ao número pequeno de diabéticos, nos grupos com tempo maior em diálise, os com, pelo menos, quatro anos foram agrupados em uma mesma categoria. $\mathrm{O}$ risco de morte foi significantemente menor em diabéticos com número maior de anos em diálise, independentemente dos efeitos das variáveis demográficas (i.e., idade, sexo e região geográfica). $O$ grupo de diabéticos com quatro ou maior número de anos em tratamento dialítico apresentou um risco de 
Figura I - Associação entre número de anos em tratamento dialítico e risco de morte, não ajustada $(\triangle)$ e ajustada ( () para idade, sexo, região geográfica e diabetes; Ref=grupo de referência ( $(-<2$ anos). A linha interrompida representa risco relativo $=1$

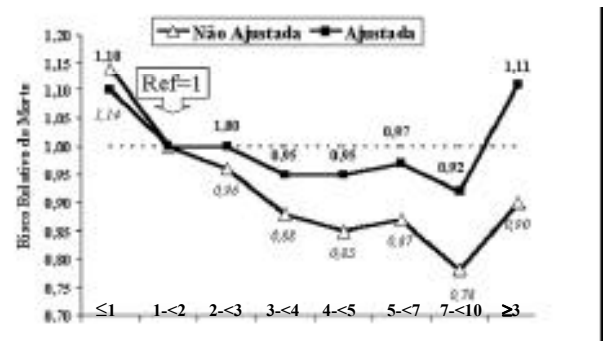

Figura 2 - Associação ajustada entre número de anos em tratamento dialítico e risco de morte em não-diabéticos $(\triangle)$ e diabéticos ( $\square)$; Ref=grupo de referência (I-<2 anos). A linha interrompida representa risco relativo $=$ I

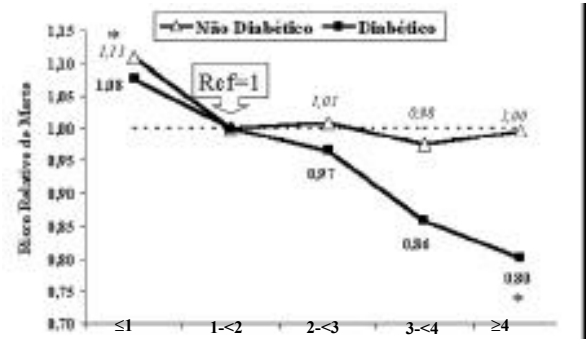

* $p<0,05$

morte $20 \%$ menor do que o grupo que estava em tratamento entre I e 2 anos $(R R=0,80$; $\mathrm{p}<0,05)$. Em não-diabéticos, o risco de morte foi II\% maior $(R R=I, I I ; p<0,05)$ nos que estavam em diálise por um período menor do que um ano, comparado com os que vinham em tratamento entre um e dois anos. Neste grupo dos não-diabéticos, no entanto, não se observou diferença significante ao compararse pacientes dialisados por, pelo menos, dois anos com os que estavam em diálise entre um e dois anos (RR entre 0,98 e I,0I).

\section{Discussão}

Os dados dos Centros de Diálise do Brasil indicam que os portadores de EFDR, que permanecem entre 3 e 10 anos em tratamento dialítico, têm um risco de morte inferior ao dos pacientes com menor número de anos em diálise. A associação entre tempo mais longo em diálise e redução do risco de morte ficou menos acentuada ao ajustar-se para o efeito da idade. A associação quase que não se alterou após a inclusão das demais variáveis demográficas, ou seja, o sexo e a região geográfica onde o paciente estava sendo dialisado. Os riscos relativos, no entanto, aproximaram-se da hipótese nula (ou seja $R R=I$ ), após a inclusão do diagnóstico de diabetes no modelo de Cox. De acordo com os resultados, o menor risco de morte em pessoas com tempo maior em diálise pode ser explicado, ao menos parcialmente, pela menor média de idade e pela menor prevalência de diabetes. Conforme mostrado em estudos prévios, idade mais avançada e diabetes melito são variáveis fortemente associadas com menor sobrevida, em pacientes em tratamento dialítico 8,9 .

Diferente do presente estudo, Chertow et al., nos Estados Unidos, não observaram associação entre anos em diálise e risco de morte na análise não ajustada ${ }^{5}$. Relataram, no entan- to, que o risco de morte aumentava em $6 \%$ para cada ano em diálise, após ajustar para diversas co-variáveis. Embora eles tenham avaliado o número de anos em tratamento dialítico como variável contínua, não deixaram claro se a relação entre o número de anos e 0 risco de morte é linear. Nossos dados mostram que, no Brasil, a associação entre número de anos em tratamento dialítico e risco de morte não pode ser vista como linear. Em um estudo utilizando dados do United States Renal Data System (USRDS), Okechukwu et al. também mostraram que a associação entre tempo em tratamento dialítico e risco de morte nãoé linear ${ }^{6}$. Eles observaram um aumento do risco de morte entre um e quatro anos em diálise. Esta tendência inicial foi seguida de redução da mortalidade 6 . No trabalho de Okechukwu et al., a associação entre tempo em tratamento dialítico e risco de morte foi, em parte, explicada por diabetes e outros fatores prognósticos que têm sido descritos em pacientes com EFDR $8,10-16$, incluindo: adesão ao tratamento, dose de diálise, nível de fósforo, hematócrito, índice de massa corpórea, doença vascular periférica, doença arterial coronariana e estado nutricional. Infelizmente, no presente estudo não foi possível avaliar as influências destes fatores potencialmente modificáveis na associação entre tempo em tratamento dialítico e risco de morte.

Nossos dados sugerem que 0 efeito do número de anos em tratamento dialítico e 0 risco de morte difere entre diabéticos e nãodiabéticos. Os diabéticos, com tempo maior em diálise, apresentaram menor risco de morte, mesmo após o ajuste para idade, sexo e região geográfica. Em não-diabéticos, o risco ajustado de morte, nos que estavam em diálise por, pelo menos, dois anos, foi similar ao observado em pacientes que estavam sendo dialisados entre um e dois anos. Os resultados observados em diabéticos diferem dos que foram descritos por Okechukwu et al. nos Estados Unidos 6 . De acordo com os dados americanos, tempo maior em diálise associase com aumento do risco de morte em diabéticos ao levar-se em consideração a idade, o sexo, variáveis laboratoriais, a dose de diálise e diversas co-morbidades. O maior número de variáveis potencialmente confundidoras ou mediadoras da associação entre tempo em tratamento dialítico e risco de morte, incluídas 
no modelo de Cox por Okechukwu et al., pode explicar, pelo menos parcialmente, a diferença de resultados entre o presente estudo brasileiro e o estudo americano.

$O$ presente estudo oferece informações que permitem um melhor entendimento sobre fatores que influenciam a mortalidade de pacientes com EFDR, tratados através diálise, no Brasil. Limitações metodológicas, no entanto, não podem ser ignoradas. A falta de informações sobre raça, fatores de tratamento e co-morbidades, além de diabetes melito, impossibilitam avaliar-se a influência destas variáveis na associação entre número de anos em tratamento dialítico e risco de morte em pacientes tratados no Brasil. Será interessante a inclusão destas variáveis em estudos futuros.

Em conclusão, o presente trabalho mostra evidência de que o risco de morte reduz-se com o aumento do número de anos que o paciente permanece em tratamento dialítico até, aproximadamente, 10 anos. Esta redução do risco de morte tende a desaparecer após se levar em consideração a idade e a presença de diabetes melito. $O$ estudo sugere que a associação entre tempo em tratamento dialítco e risco de morte difere entre paciente com e sem diabetes melito. Diferenças observadas entre os dados dos centros de diálise do Brasil e os dos Estados Unidos podem ser, pelo menos em parte, devido a um maior número de variáveis incluídas nas análises dos estudos americanos. É importante incluir dados sobre variáveis de tratamento e outras co-morbidades, além de diabetes, em investigações futuras sobre a associação entre número de anos em tratamento dialítico e risco de morte, em pacientes dos centros de diálise do Brasil. De acordo com os nossos achados, a variável "número de anos em tratamento dialítico" deve ser tratada como potencialmente confundidora em estudos que exploram fatores relacionados com risco de morte em pacientes com EFDR. O ajuste para esta variável é particularmente aplicável para estudos de coorte que avaliam fatores prognósticos em pacientes que começaram tratamento dialítico meses ou anos antes do inicio do período de acompanhamento.

\section{Agradecimento}

Nossos agradecimentos para o sr. Luiz Inácio C. Cincurá de Andrade e para o dr. Gabriel Nery pela revisão do manuscrito.

\section{SUMMARY}

Number of years ON CHRONIC DIALYSIS AND THE MORTALITY RISK IN PATIENTS WITH AND WITHOUT DIABETES MELLITUS

OBJECTIVE: To assess whether, in Brazil, the number of years on dialysis treatment in end-stage renal disease (ESRD) patients, with and without diabetes mellitus, relates to mortality risk.

METHODS: Analysis of data from the Ministry of Health of Brazil of 25.952 patients older than 18 years who were on dialytic treatment in April 1997. Death cases that occurred from April 1997 to July 2000 were recorded. Cox models were used to estimate the relative risk (RR) of the association between years on dialysis and mortality risk.

RESULTS: The mortality risk was significantly lower in patients on dialysis between 3 and $<10$ years as compared with 1 to $<2$ years (RR between 0.78 and 0.88 ). With adjustments for age and diabetes the effect of years on dialysis was reduced. In diabetics $(n=2.952)$ the adjusted mortality risk showed a trend to decrease with more years on dialysis.

Conclusions: In Brazil, the mortality risk for ESRD patients decreases with the increasing number of years on dialysis up to approximately 10 years. This finding can be partially explained by the lower mean age and prevalence of diabetes mellitus in patients with more years on dialysis treatment. The effect of years on dialysis on the mortality risk seems to be stronger in patients with diabetes. [Rev Assoc Med Bras 2003; 49(3): 266-9]

KEY wORDs: Dialysis. Mortality Risk. End-Stage Renal Disease.

\section{REFERÊNCIAS}

I. Port FK. Morbidity and mortality in dialysis patients. Kidney Int 1994; 46: I 728-37.

2. Port FK, Orzol SM, Held PJ, Wolfe RA. Trends in treatment and survival for hemodialysis patients in the United States. Am J Kidney Dis 1998; 32(Suppl 4):S34-S8.

3. Port FK. Mortality and causes of death in patients with end-stage renal failure. Am J Kidney Dis 1990; 15:215-7.

4. Held PJ, Levin NW, Bovbjerg RR, Pauly MV, Diamond LH. Mortality and duration of hemodialysis treatment. JAMA 199I; 265:87I-5.

5. Chertow GM, Johansen KL, Lew N, Lazarus JM, Lowrie EG. Vintage, nutritional status, and survival in hemodialysis patients. Kidney Int 2000; 57: I |76-8I.

6. Okechukwu CN, Lopes AA, StackAG, Feng S, Wolfe RA, Port FK. Impact of years of dialysis therapy on mortality risk and the characteristics of longer term dialysis survivors. Am J Kidney Dis 2002; 39:533-8.

7. Norusis MJ. SPSS for windows: advanced statistics, release 6.0. Chicago: SPSS Inc; 1993.

8. Mailloux LU, Bellucci AG, Mossey RT, et al. Predictors of survival in patients undergoing dialysis. Am J Med 1 988; 84:855-62.

9. Wolfe RA, Port FK. Good news, bad news for diabetic versus nondiabetic end-stage renal disease: incidence and mortality. ASAIO J 1999; 45: 117-8.

10. MaJZ, Ebben J, Xia H, Collins AJ. Hematocrit level and associated mortality in hemodialysis patients. J Am Soc Nephrol 1999; 10:6 10-9.

I I. Charra B, Calemard M, Laurent G. Importance of treatment time and blood pressure control in achieving long-term survival on dialysis. Am J Nephrol 1996; 16:35-44.

12. Block GA, Hulbert-Shearon TE, Levin NW, PortFK. Association of serum phosphorus and calcium $x$ phosphate product with mortality risk in chronic hemodialysis patients: a national study. Am J Kidney Dis 1998; 31:607- 7.

13. Combe C, Chauveau P, Laville M, Azar R, Cano N, Canaud B et al. Influence of nutritional factors and hemodialysis adequacy on the survival of I,610 French patients. Am J Kidney Dis 200I; 37(Suppl 2):S8I-S8.

14. Held PJ, Port FK, Wolfe RA, Stannard DC, Carroll CE, Daugirdas JT et al. The dose of hemodialysis and patient mortality. Kidney Int 1996; 50:550-6.

15. Mailloux LU, Napolitano B, Bellucci AG, Mossey RT, Vernace MA, Wilkes BM. The impact of co-morbid risk factors at the start of dialysis upon the survival of ESRD patients. ASAIO J 1996; 42: 164-9.

16. Owen WF, Lew NL, Liu Y, Lowrie EG, Lazarus JM. The urea reduction ratio and serum albumin concentration as predictors of mortality in patients undergoing hemodialysis. N Engl J Med I 993; 329: 100 I-6.

Artigo recebido:01/07/02

Aceito para publicação: 02/09/02 